\title{
CADASIL Initially Presented with a Seizure
}

\section{Case Report}

Journal of Epilepsy Research pISSN 2233-6249 / eISSN 2233-6257

Received October 30, 2016

Accepted November 26, 2016

Corresponding author: Jay Chol Choi

Departments of Neurology, Jeju National University Hospital, Aran 13 gil 15, Jeju, Jeju Special Self-Governing Province 63241, Korea

Tel. $+82-64-754-8160$

Fax. + 82-64-717-1131

E-mail; jaychoi@jejunu.ac.kr

\author{
Jung-Hwan Oh ${ }^{1}$, Bong Su Kang ${ }^{2}$, Jay Chol Choi \\ ${ }^{1}$ Department of Neurology, Jeju National University School of Medicine, Jeju; \\ ${ }^{2}$ Yangpyeong Hospital, Yangpyeong, Korea
}

Cerebral autosomal dominant arteriopathy with subcortical infarcts and leukoencephalopathy (CADASIL) is a hereditary disease of the cerebral small blood vessels characterized by recurrent ischemic strokes, migraine, and progressive cognitive impairment. In patients with CADASIL, in whom subcortical white matter structures are typically involved, epileptic seizures have been rarely reported as an initial clinical symptom. We describe a patient genetically confirmed as having CADASIL who initially presented with a seizure. (2016;6:106-108)

Key words: CADASIL, Seizures, White matter

\section{Introduction}

Cerebral autosomal dominant arteriopathy with subcortical infarcts and leukoencephalopathy (CADASIL) is a hereditary disease of the cerebral small blood vessels caused by mutations in the Notch 3 gene. ${ }^{1}$ The main clinical manifestations are recurrent ischemic strokes, migraine, and progressive cognitive impairment. ${ }^{1}$ Epileptic seizures have only been reported in $5-11 \%$ of patients with CADASIL. ${ }^{2,3}$ Moreover, the occurrence of a seizure is very rare as an initial manifestation in patients with CADASIL., ${ }^{2,3}$ Here, we describe a patient with CADASIL who initially presented with a seizure.

\section{Case}

A 43-year-old male patient visited the emergency department because of a new-onset seizure. According to the witness, the patient was talking on the phone to his office staff, and then he suddenly screamed and fell on the floor. Soon he developed a generalized tonic-clonic seizure for about 3 minutes followed by postictal confusion that lasted several seconds. He denied a history of febrile convulsion and an infection involving the central nervous system. He was a current smoker and had hypercholesterolemia, but he did not experience a transient ischemic attack, recurrent headache, or psychiatric illness. His family history showed that his father died of stroke, and his mother had epilepsy due to neurocysticercosis. A neurological examination performed in the emergency department was normal. The serum glucose level increased up to $164 \mathrm{mg} / \mathrm{dL}$; otherwise, there were no other abnormal laboratory findings.

A 1.5-T brain magnetic resonance imaging (MRI) scan showed multiple white matter hyperintensity lesions on both periventricular white matter, anterior temporal lobes and external capsules (Fig. 1). A scalp electroencephalogram showed frequent focal spikes over the left frontal area and secondary bilateral synchrony (Fig. 2). Results of the genetic test showed that the patient had a heterozygote R544C mutation at exon 11 of the Notch 3 gene.

Initially, oxcarbazepine (600mg daily) was given to the patient for seizure control. However, the patient complained of headache after the intake of oxcarbazepine. Therefore, topiramate (200mg daily) was used instead of oxcarbazepine. The patient had been seizure-free after antiepileptic drug treatment during the 5-year follow-up.

\section{Discussion}

Seizures are uncommon manifestations in patients with CADASIL., They usually occur following a stroke, and they are often characterized by generalized tonic-clonic seizures in patients with CADASIL. In a report of 102 patients with CADASIL, $10 \%$ of them had seizures. Most of these patients had generalized seizures (9 of 10 patients), and they had a history of previous stroke; however, none of them had a seizure as the initial manifestation. ${ }^{3}$ A pooled data analysis of CADASIL demonstrated that 6 of 105 patients experienced seizures, and in only 3 of them, seizures occurred as an initial manifestation. ${ }^{2}$ Recently, two patients with CADASIL who initially presented with 

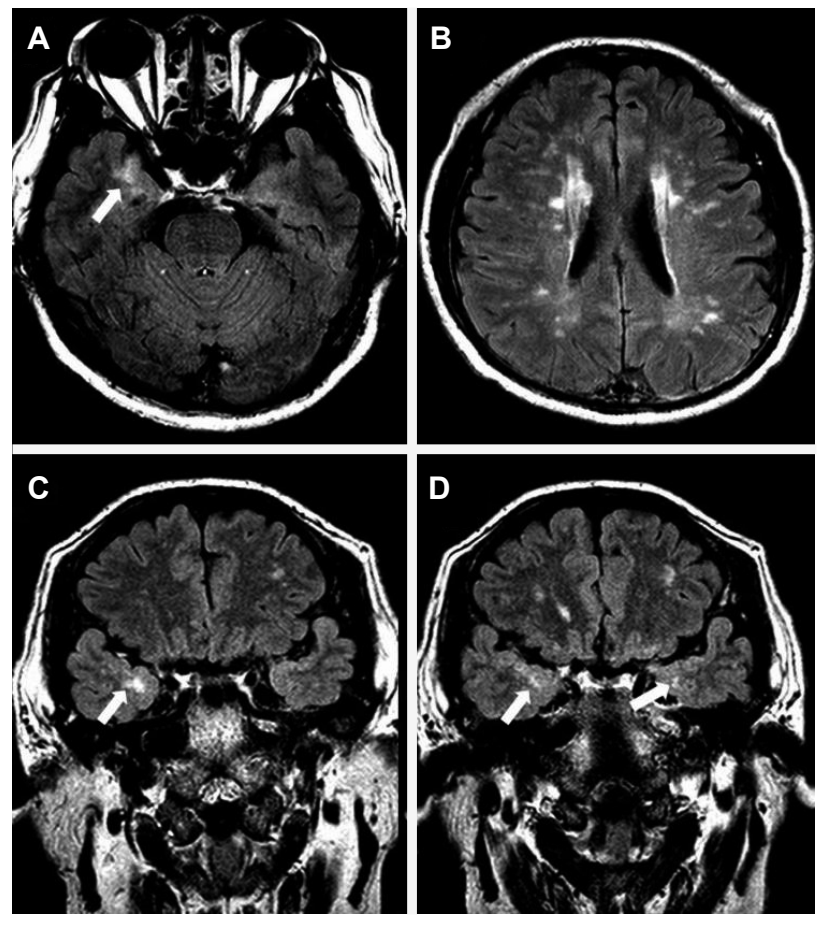

Figure 1. Brain fluid-attenuated inversion recovery magnetic resonance imaging scans. Multiple white matter hyperintensity lesions are shown in the periventricular white matter, anterior temporal lobes (arrows) and external capsules on axial (A, B) and coronal (C, D) images.

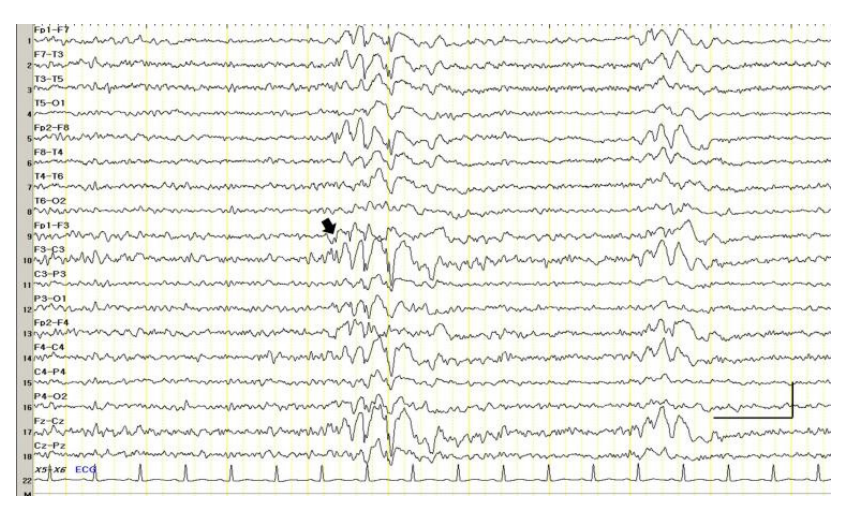

Figure 2. Longitudinal bipolar electroencephalogram showing focal spikes over the left fontal region (maximum at F3; arrow) followed by a generalized synchronous spike and slow waves (secondary bilateral synchrony). Vertical bar $=100 \mathrm{uV}$; horizontal bar $=1 \mathrm{sec}$.

seizures were reported. ${ }^{4,5} \mathrm{~A}$ focal seizure arising from the parieto-occipital junction was reported in one case. ${ }^{4}$ In the other case, focal status epilepticus of left hemispheric origin was reported. ${ }^{5}$ However, in either of these reports, the mechanism of seizures associated with CADASIL was not mentioned.

Although the exact cause of an epileptic seizure in patients with CADASIL is currently unknown, high-signal-intensity lesions fre- quently found on anterior temporal regions may be associated with epileptogenesis in these patients. Patients with temporal lobe epilepsy (TLE) sometimes have white matter abnormalities in the anterior temporal lobe (WAATL), which are also frequently observed in patients with CADASIL., ${ }^{3,6}$ WAATL are clinically useful because they indicate the side of the seizure foci in patients with TLE. ${ }^{6,7}$

In a post-mortem high-resolution 7-T MRI study, a large number of cortical lesions and subcortical lesions were also shown in patients with CADASIL. ${ }^{8}$ Recently, Gasparini et al. suggested a noteworthy hypothesis on the association between leukoaraiosis and epileptic seizures. They asserted that occult cortical micro-infarcts may be associated with epilepsy in patients with leukoaraiosis. ${ }^{7}$ Leukoaraiosis is commonly observed on MRI scans of patients with CADASIL; ${ }^{9}$ hence, epileptogenic cortical lesions can lead to seizures in patients with CADASIL. Unfortunately, high-resolution 7-T MRI could not be performed to identify cortical lesions in the present patient, because it is currently unavailable at our clinic.

In some previous reports, cholinergic denervation in the cerebral cortex and white matter tract has been observed in patients with CADASIL. ${ }^{10}$ Cholinergic neurons modulate excitability in the central nervous system. ${ }^{11}$ Additionally, several experimental studies have demonstrated that cholinergic denervation may generate seizures through the facilitation of kindling. ${ }^{11}$ Interestingly, Keverne et al. reported cholinergic neuronal damage along white matter tracts that mainly reached toward the frontal cortices in nine patients with CADASIL. ${ }^{12}$ Consequently, susceptibility to seizures may be increased in patients with CADASIL, as white matter lesions in the frontal lobe seem more prone to epileptic seizures than those in the temporal lobe. Therefore, our patient's seizure could have originated from the frontal region despite involvement of the temporal white matter.

Although seizures are uncommon and likely to occur with a stroke in patients with CADASIL, patients with this disease can initially present with a seizure. When physicians encounter patients with new-onset seizures with an unexplained white matter lesion on a brain MRI scan, they should consider CADASIL. In addition, the exact mechanism of seizure development is still unclear, so further research with more patients with CADASIL is warranted.

\section{References}

1. Choi JC, Kang SY, Kang JH, Park JK. Intracerebral hemorrhages in CADASIL. Neurology 2006;67:2042-4.

2. Desmond DW, Moroney JT, Lynch T, Chan S, Chin SS, Mohr JP. The 
natural history of CADASIL: a pooled analysis of previously published cases. Stroke 1999;30:1230-3.

3. Dichgans $M$, Mayer $M$, Uttner $l$, et al. The phenotypic spectrum of CADASIL: clinical findings in 102 cases. Ann Neurol 1998;44:731-9.

4. Velizarova R, Mourand I, Serafini A, Crespel A, Gelisse P. Focal epilepsy as first symptom in CADASIL. Seizure 2011;20:502-4.

5. Haddad N, Ikard C, Hiatt K, Shanmugam V, Schmidley J. Recurrent status epilepticus as the primary neurological manifestation of CADASIL: A case report. Epilepsy Behav Case Rep 2015;3:26-9.

6. Adachi Y, Yagishita A, Arai N. White matter abnormalities in the anterior temporal lobe suggest the side of the seizure foci in temporal lobe epilepsy. Neuroradiology 2006;48:460-4.

7. Gasparini S, Ferlazzo E, Beghi E, et al. Epilepsy associated with Leukoaraiosis mainly affects temporal lobe: a casual or causal relationship? Epilepsy Res 2015;109:1-8.
8. Jouvent E, Poupon C, Gray $F$, et al. Intracortical infarcts in small vessel disease: a combined 7-T postmortem MRI and neuropathological case study in cerebral autosomal-dominant arteriopathy with subcortical infarcts and leukoencephalopathy. Stroke 2011;42:e27-30.

9. Abe K, Murakami T, Matsubara E, Manabe Y, Nagano I, Shoji M. Clinical Features of CADASIL. Ann N Y Acad Sci 2002;977:266-72.

10. Manganelli F, Ragno M, Cacchio $G$, et al. Motor cortex cholinergic dysfunction in CADASIL: a transcranial magnetic demonstration. Clin Neurophysiol 2008;119:351-5.

11. Silveira DC, Holmes GL, Schachter SC, Geula C, Schomer DL. Increased susceptibility to generalized seizures after immunolesions of the basal forebrain cholinergic neurons in rats. Brain Res 2000;878:223-7.

12. Keverne JS, Low WC, Ziabreva I, Court JA, Oakley AE, Kalaria RN. Cholinergic neuronal deficits in CADASIL. Stroke 2007;38:188-91. 